\title{
Dexamethasone Aggravates Hippocampal Apoptosis and Learning Deficiency in Pneumococcal Meningitis in Infant Rats
}

\author{
STEPHEN L. LEIB, CHRIS HEIMGARTNER, YOENG-DELPHINE BIFRARE, \\ JUTTA M. LOEFFLER, AND MARTIN G. TÄUBER \\ Institute for Infectious Diseases, University of Bern, CH-3010 Bern, Switzerland
}

\begin{abstract}
ABST
In an infant rat model of pneumococcal meningitis the effect
of dexamethasone on neuronal injury in the hippocampus and on
learning disability after recovery from the disease was examined.
Treatment with dexamethasone or vehicle was started $18 \mathrm{~h}$ after
infection, concomitant with antibiotics. Neuronal apoptosis in the
hippocampal dentate gyrus 34 h after infection was significantly
aggravated by dexamethasone treatment compared with vehicle
controls ( $p=0.02)$. Three weeks after acute pneumococcal
meningitis, learning capacity of animals was assessed in the
Morris water maze. The results showed a significantly impaired
learning performance of infected animals treated with dexameth-
asone compared with vehicle controls $(p=0.01)$. Dexametha-
\end{abstract}
Inflammation in the subarachnoid and ventricular space is the hallmark of bacterial meningitis and appears to be responsible for much of the pathophysiologic consequences of the disease (1-3). Therapy with highly active antibiotics is only partially effective in preventing death and the development of neurologic sequelae in patients with bacterial meningitis $(4,5)$. Even with optimal treatment, mortality remains high in certain patient groups and up to $40 \%$ of the survivors of meningitis suffer from neurologic sequelae, including sensory-motor deficits, cerebral palsy, seizure disorders, mental retardation, and learning impairments $(6-8)$.

Given the harmful effect of the inflammatory reaction, antiinflammatory adjuvant treatment with dexamethasone has been tested in several controlled clinical trials (9-11). A recent meta-analysis concluded that the drug is effective in preventing hearing loss in H. influenzae meningitis and has some beneficial effects on hearing and neurologic sequelae in pneumococcal meningitis when given before or at the time of the first

Received September 25, 2002; accepted January 16, 2003.

Correspondence: Stephen L. Leib, M.D., Institute for Infectious Diseases, University of Bern, Friedbühlstrasse 51, 3010 Bern, Switzerland; e-mail: stephen.leib@ifik.unibe.ch

This work was supported by grants from the Swiss National Science Foundation (32-61654.00 and 632-66057.01), the NIH (NS-35902), and the Meningitis Research Foundation (Grant 14/00).

DOI: 10.1203/01.PDR.0000079185.67878.72 sone had no effect on hippocampal injury or learning in uninfected controls. Thus, dexamethasone as adjuvant therapy increased hippocampal cell injury and reduced learning capacity in this model of pneumococcal meningitis in infant rats. (Pediatr Res 54: 353-357, 2003)
CSF, cerebrospinal fluid
Abbreviations
DG, dentate gyrus
MMP, matrix metalloproteinase
PBN, alpha- phenyl-tert-butyl nitrone
TACE, tumor necrosis factor alpha converting enzyme

antibiotic dose (11). However, in most of the individual trials, these benefits were either marginal or could not be detected at all (12). Furthermore, H. influenzae meningitis has virtually disappeared in countries that have implemented programs with conjugated vaccines in infants and children (13). Consequently, the use of dexamethasone has remained controversial $(12,14,15)$.

Two brain structures prominently affected in bacterial meningitis are the cortex and the hippocampus. Neuronal apoptosis in the hippocampal dentate gyrus has been observed in humans dying from bacterial meningitis (16) and in animal models of meningitis (17-20). Neuropsychological evidence in humans and animal models suggests that damage to the hippocampus impairs learning and spatial memory (21-23). We have recently shown that the extent of learning dysfunction is related to the extent of hippocampal apoptosis in infant rats with pneumococcal meningitis $(17,19)$

A study in adult rabbits with pneumococcal meningitis has suggested that the number of neurons undergoing apoptosis in the hippocampal formation was increased when antibiotic treatment was combined with dexamethasone therapy (18). However, the outcome of adjuvant dexamethasone in a model of infant meningitis and the effect on learning dysfunction as a consequence of bacterial meningitis has not been studied to date. Together with the questions surrounding the benefits of 
dexamethasone in patients with bacterial meningitis, this prompted us to study the effect of the drug in our model of pneumococcal meningitis in infant rats. Specifically, we examined whether dexamethasone influenced apoptotic injury in the dentate gyrus and whether this was associated with an effect on learning capacity after recovery from acute meningitis.

\section{MATERIALS AND METHODS}

Model of meningitis. The animal studies were approved by the Animal Care and Experimentation Committee of the Canton of Bern, Switzerland, and followed National Institutes of Health guidelines for the performance of animal experiments. Nursing Sprague-Dawley rat pups were infected on postnatal day $11(n=142)$ by intracisternal injection with $10 \mu \mathrm{L}$ of saline containing an inoculum of $\log _{10} 6.5 \pm 0.6 \mathrm{cfu} / \mathrm{mL}$ Streptococcus pneumoniae $(17,19,24,25)$. Uninfected animals $(n=62)$ were injected intracisternally with $10 \mu \mathrm{L}$ of sterile, pyrogen free saline. At $18 \mathrm{~h}$ after infection, animals were weighed and assessed clinically. To document meningitis, $10 \mu \mathrm{L}$ of cerebrospinal fluid was obtained by puncture of the cisterna magna and cultured quantitatively.

Treatment. All infected animals $(n=142)$ received antibiotic treatment (ceftriaxone $100 \mathrm{mg} / \mathrm{kg}$ s.c. bid; Roche Pharma, Reinach, Switzerland) following CSF collection at $18 \mathrm{~h}$. Antibiotic treatment was continued for 2 doses in infected animals subsequently examined histopathologically $(n=38)$ and for $5 \mathrm{~d}$ in animals subjected to learning testing $(n=104)$. Animals to be assessed histopathologically ( $n=38$ for meningitis; $n=$ 14 for uninfected controls) were randomized for treatment with dexamethasone $(0.7 \mathrm{mg} / \mathrm{kg}$ s.c. $\mathrm{q} 8 \mathrm{~h}$ from 18 to $34 \mathrm{~h}$ after infection; $n=16$ for meningitis; $n=8$ for controls) or vehicle ( $n=22$ for meningitis; $n=6$ for controls). Animals to be assessed for learning performance were randomized for treatment with dexamethasone $(0.7 \mathrm{mg} / \mathrm{kg}$ s.c. q $8 \mathrm{~h}$ for $4 \mathrm{~d} ; n=34)$ or vehicle $(n=70)$ concomitant with the first antibiotic dose. Data presented here combine two separate experiments using the same protocol. In the first trial, normal saline was administered as vehicle to control animals (infected controls, $n=31$; uninfected controls, $n=31$ ). In the second trial, control animals received PBS containing $1 \%$ Tween $80(0.2 \mathrm{~mL}$ s.c. $)$ as vehicle (infected controls, $n=52$; uninfected controls, $n=$ 32) because in this trial another drug was tested concomitantly with dexamethasone, which had to be dissolved in PBS/Tween (17). Treatment with $\mathrm{NaCl}$ versus $\mathrm{PBS} / \mathrm{Tween}$ did not result in significant differences in hippocampal histopathology or learning performance either in uninfected or in infected control animals. However, for both the infected and uninfected control group, animals treated with $\mathrm{NaCl}$ or $\mathrm{PBS} /$ Tween had to be combined to make the differences between the three treatments (uninfected; infected vehicle treated; infected

dexamethasone treated) statistically significant.

Effect of treatment on bacterial killing in CSF. In eleven infected animals (dexamethasone, $n=5$; PBS/Tween, $n=6$ ) repeated cisternal punctures were performed at 18,22 , and $30 \mathrm{~h}$ post-infection. CSF bacterial titers were determined quantitatively by serial dilution to assess the influence of dexamethasone therapy on the decline of bacterial titers in CSF following initiation of antibiotic therapy.

Histopathology. At $34 \mathrm{~h}$ post-inoculation, infected animals treated with dexamethasone $(n=10)$, uninfected animals treated with dexamethasone $(n=8)$ and infected $(n=19)$ and uninfected controls $(n=6)$ were killed with an overdose of pentobarbital. Animals dying spontaneously before $34 \mathrm{~h}(n=$ 9) were not evaluated. Immediately after euthanasia, animals were perfused via the left ventricle with $15 \mathrm{~mL}$ of $4 \%$ paraformaldehyde in PBS ( $\mathrm{pH}$ 7.4). Brains were removed, postfixed and snap-frozen at $-60^{\circ} \mathrm{C}$ in methylbuthane and cut at 45-60 $\mu \mathrm{m}$ intervals on a cryotome to obtain four coronal sections of the hippocampal region. Sections were mounted on gelatinized glass slides for staining. After dehydration, sections were Nissl stained with cresyl violet and coverslips were fixed with Entellan ${ }^{\circledR}$ (Merck, Darmstadt, Germany). Neuronal injury in the dentate gyrus was evaluated as described previously (24). Apoptosis in the granule cell layer of the hippocampus, defined as cells showing markedly shrunken, condensed or fragmented nuclei (Fig. 1) was counted at 400x in 3 visual fields for each of the four blades of the dentate gyrus. An average per dentate gyrus (six visual fields) per animal was
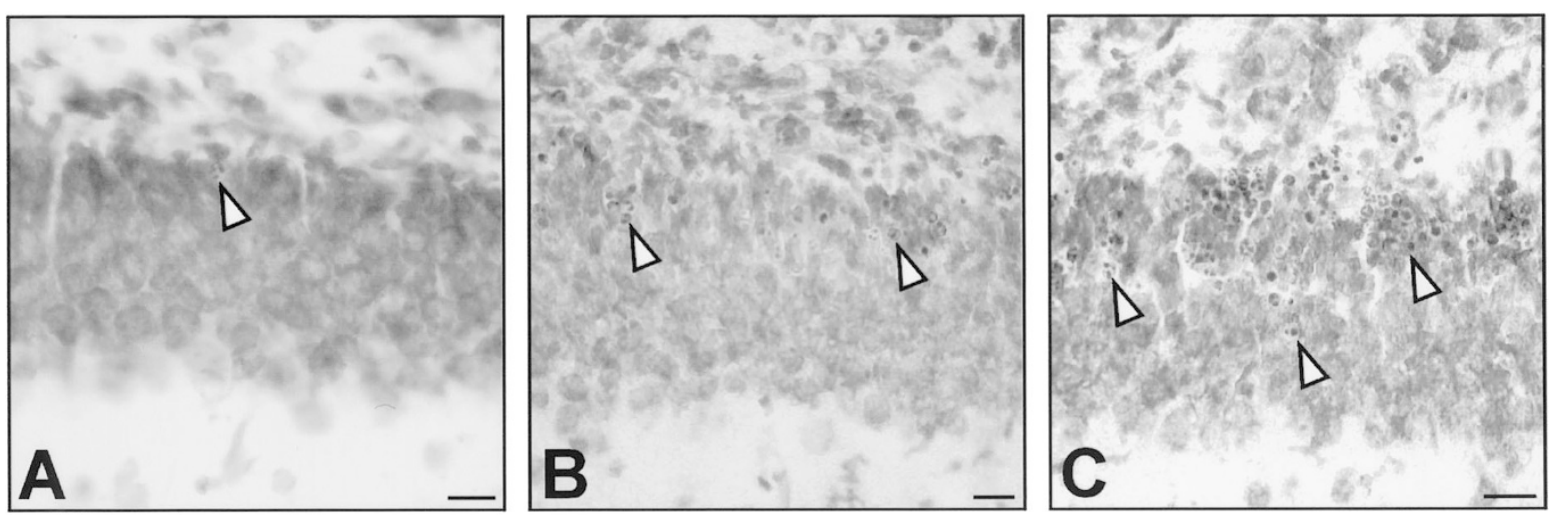

Figure 1. Hippocampal dentate gyrus histology of infant rats suffering from pneumococcal meningitis at $34 \mathrm{~h}$ after infection. $A$ ) In the dentate gyrus of uninfected controls physiologic occurrence of neuronal apoptosis is sporadically visible by the formation of condensed and fragmented nuclei (arrowhead). $B$ ) In infected vehicle treated rats formation of apoptotic bodies is characteristically observed in the inner rim of the dentate gyrus (arrowheads) at $34 \mathrm{~h}$ after infection. C) Treatment with dexamethasone markedly increased the occurrence of apoptotic bodies (arrowheads) in the hippocampal dentate gyrus. Cresyl violet; original magnification $\times 300$; bar $50 \mu \mathrm{m}$. 
calculated from all slides evaluated. Histopathologic evaluations were done by an investigator blinded to the clinical, microbiologic and treatment data of the animals.

Assessment of learning function. Testing of spacial learning and memory based on visual clues was performed in the Morris water maze, as previously described $(17,19)$. Before the test, gross vestibulomotor dysfunction was excluded using a rotating rod, as described $(17,19)$. For assessment of learning function, time and distance to reach a submersed, for the rats invisible platform was determined by registering the swim patterns with the video tracking system Ethovision ${ }^{\circledR}$ (Noldus Information Technology, Wageningen, Netherlands).

Thirty-two day old survivors of meningitis $(n=85 ; 19 / 104$ infected animals died or had to be killed for ethical reasons before the age of $32 \mathrm{~d})$ treated with dexamethasone $(n=28)$ or vehicle $(n=57)$ and uninfected control animals $(n=48)$ were transferred to the test room where they were given $24 \mathrm{~h}$ to acclimatize in 12 hourly light/dark cycles. Animals were provided with water and food ad libitum. From day 1 to 4 animals performed five training trials per day with the invisible platform in a fixed position throughout the test. The rats were put into the water at randomly assigned entry points with their head directed toward the wall of the tank. If an animal found the platform within $90 \mathrm{~s}$, it was allowed to stay on it for $15 \mathrm{~s}$ before it was put back to the cage. Otherwise the rat was guided to the platform by hand and was allowed to stay on it for $15 \mathrm{~s}$. Between trials animals rested $45 \mathrm{~min}$.

Statistical methods. Normally distributed variables were expressed as mean $\pm \mathrm{SD}$. Differences between groups were analyzed by $t$ test or ANOVA and the Newman-Keuls posthoc test for multiple comparison in the case of multiple groups. Survival curves were analyzed using the Kaplan-Meier method. Incidence of seizures, spontaneous death and cortical injury were compared using Fisher's exact test. Hippocampal apoptosis was analyzed with the Mann-Whitney rank-sum test. Distances moved in the water maze task were compared with repeated-measures ANOVA, and pair wise comparison was done with the Tukey-Kramer adjustment. SAS Version 8.0 (SAS Institute Inc., Cary, NC, U.S.A.) software was used.

\section{RESULTS}

Characteristics of disease. By $18 \mathrm{~h}$ after infection, meningitis was documented in all infected animals by positive CSF cultures for Streptococcus pneumoniae. Animals subsequently treated with dexamethasone and infected control animals had similar bacterial titers in CSF $\left(\log _{10} 7.7 \pm 0.6\right.$ versus $7.7 \pm 0.7$ $\mathrm{cfu} / \mathrm{mL}, p=$ n.s.). The decline of CSF titers after the first dose of ceftriaxone in animals treated with dexamethasone versus controls was similar at $22 \mathrm{~h}\left(\log _{10} 3.4 \pm 2.5\right.$ versus $3.4 \pm 3.0$; $p=$ n.s.) and $30 \mathrm{~h}$ (sterile in both groups). Spontaneous death occurred in $37 \%$ of dexamethasone treated animals and in 35\% of infected controls ( $p=$ n.s.). Seizures were observed with the same frequency in infected controls (15\%) and dexamethasone treated animals $(14 \% ; p=$ n.s.). The known effect of dexamethasone therapy on catabolism was reflected in a decreased weight at the age of $32 \mathrm{~d}$, when surviving animals were tested for motor and learning function. Dexamethasone treated ani- mals weighted significant less than vehicle treated animals both among the infected $(90.1 \pm 11.1 \mathrm{~g}$ versus $112.8 \pm 12.2 \mathrm{~g} ; p<$ $0.001)$ and uninfected groups $(68.0 \pm 7.6 \mathrm{~g}$ versus $120.7 \pm$ $15.8 \mathrm{~g} ; p<0.001)$ (26). No weight differences were present at the time of infection (data not shown).

Histopathology. Previous studies have shown that in this model of bacterial meningitis neuronal cells in the subgranular zone of the dentate gyrus undergo apoptosis $(17,19)$. In the present study, apoptosis in the dentate gyrus was more frequent in the infected dexamethasone treated animals than in infected controls (median [range] 56.6 [2.63-132.1] versus 5.5 [0-31.9], $p<0.01$ ) (Fig. 1 and 2). In uninfected animals, there was a slight, nonsignificant increase in apoptosis in the hippocampus in animals treated with dexamethasone compared with controls (Fig. 2). Injury to the cortex was relatively minor, such that the previously documented beneficial effect of dexamethasone on this form of injury could not be evaluated in the present study (27).

Learning assessment. All animals had normal vestibulomotor function based on their ability to stay on the rotating rod. The distance to reach the platform decreased in all groups of animals over the four test days $(p<0.001)$. Uninfected controls improved their learning significantly faster than infected control animals $(p<0.001)$ (Fig. 3). Furthermore, among infected animals, those treated with dexamethasone had significantly reduced learning capacity compared with infected controls ( $p=0.01$; Fig. 3). Among the uninfected controls, there was no measurable effect of dexamethasone treatment on performance in the water maze learning test (data not shown).

\section{DISCUSSION}

In the present study, we found that dexamethasone, which has been shown to have some beneficial effects as adjuvant

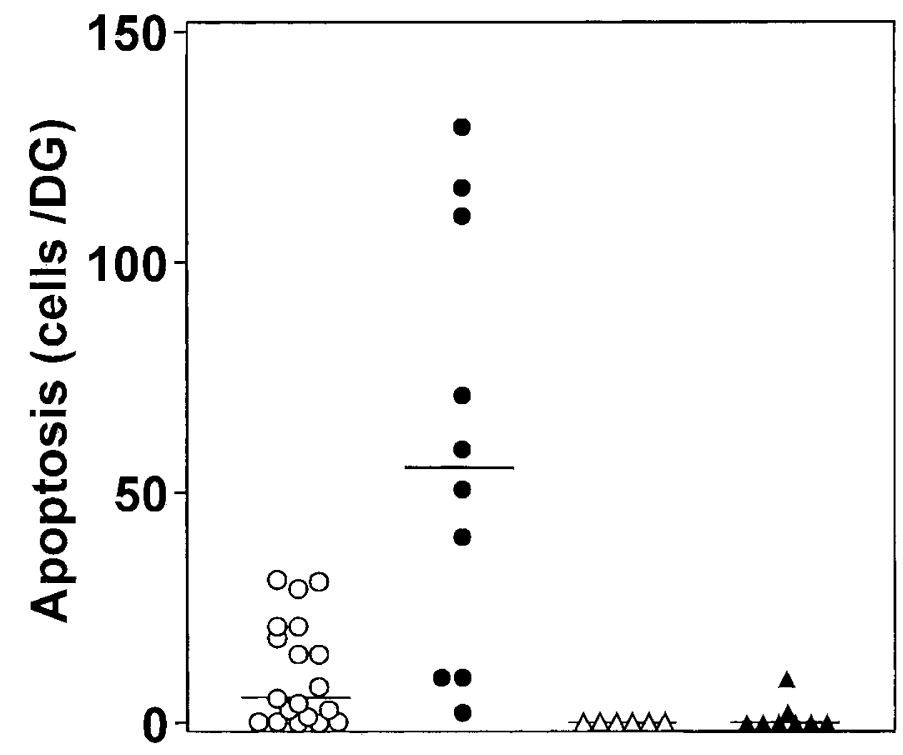

Figure 2. Effect of dexamethasone on hippocampal apoptosis in infant rats with pneumococcal meningitis. Dexamethasone significantly increased apoptosis in infected animals ( $p<0.01$, Mann-Whitney rank-sum test). In uninfected animals no effect was observed. (, infected vehicle treated; $\bigcirc$, infected dexamethasone treated; $\triangle$, uninfected vehicle treated; $\boldsymbol{\Delta}$, uninfected dexamethasone treated). 


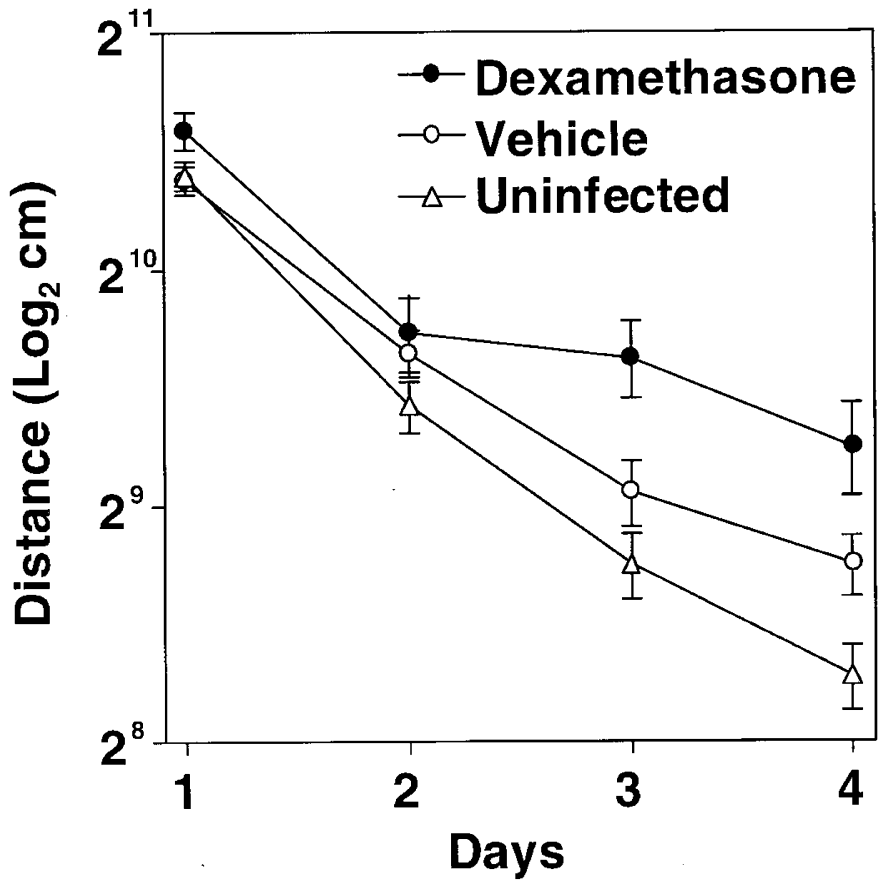

Figure 3. Assessment of learning capacity by Morris water maze $3 \mathrm{wk}$ after infection (analysis of the distance to reach platform. Uninfected control animals learned significantly faster than infected animals $(p<0.001)$. Adjuvant therapy of meningitis with dexamethasone led to inferior performance compared with vehicle treated animals $(p=0.01)$. ANOVA, pair wise comparison with Tukey-Kramer adjustment.

therapy in bacterial meningitis $(9,11,28)$, led to an increase in apoptotic cell death in the hippocampal dentate gyrus of infant rats with pneumococcal meningitis. These findings expand on the previous observation in adult rabbits with pneumococcal meningitis, where dexamethasone also led to an increase in hippocampal neuronal apoptosis (18). Thus, in two different experimental systems, infant rats and adult rabbits, there is an association between dexamethasone treatment and hippocampal neuronal apoptosis. More importantly, the present study demonstrates that the increase in hippocampal apoptosis induced by adjuvant therapy with dexamethasone was associated with reduced learning capacity in the Morris water maze test, which assesses spacial learning and memory based on visual cues $(17,19)$.

The results of the present study are similar to a previous study from our laboratory, in which the brain-penetrating antioxidant PBN also increased dentate gyrus apoptosis and at the same time reduced performance in the water maze test (17, 19). Studies using different experimental probes with different molecular mechanisms strengthen the contention that apoptotic neuronal death in the dentate gyrus has a negative effect on spacial learning in experimental pneumococcal meningitis and suggest that the details of this association warrant further studies.

Neurons of the hippocampal regions show a high density of glucocorticoid receptors (29) and there are complex interactions between systemic corticosteroid levels and cell viability of hippocampal neurons (30). Recent studies indicate that stimulation of the high affinity mineralocorticoid receptor shifts the balance between pro- and anti-apoptotic factors of the $b c l-2$ gene family in favor of cell survival, while stimulation of the low affinity glucocorticoid receptor has the opposite effect (30). In keeping with these observations, treatment of rats with the glucocorticoid dexamethasone induced apoptosis in the hippocampus, especially in the dentate gyrus and CA1 and CA3 regions $(31,32)$. Also, chronic corticosteroid (prednisone) treatment in patients with systemic disease in a retrospective study appeared to deteriorate hippocampus-dependent explicit memory (33).

Apoptotic cell death in the dentate gyrus in experimental pneumococcal occurs in a caspase-3 dependent fashion (34, 35). However, little is known about the stimuli that lead to the induction of this cell death pathway. Among various adjuvant therapies tested in the pneumococcal meningitis model in infant rats, only the use of a combined MMP/TACE inhibitor has reduced the extent of apoptotic injury and the associated impaired performance in the Morris water maze (17).

The basis for the adverse effects of dexamethasone remains currently unclear. Importantly, administration of dexamethasone in the dosing schedule used in this study had no effect on hippocampal cell death or functional performance in uninfected animals. Thus, the adverse effect of the drug was only unmasked by the presence of meningitis. This could indicate an increased susceptibility of dentate gyrus neurons to the effects of steroids during meningitis. The present observations raise, in addition to questions regarding the molecular mechanisms, several important clinical questions. Prominently, efforts should be made to explore the possibility that dexamethasone has similar adverse effects on hippocampal structure and function in humans with bacterial meningitis treated with dexamethasone. While the controlled clinical studies have made attempts to assess neurologic outcome, the methods used for this assessment and the follow-up time may not have been sufficient to reliably detect more subtle effects of corticosteroids on hippocampal functions. It is also not known whether the observed effect is age-dependent. In rodents, older animals appeared to be more susceptible to steroid-induced apoptosis, but no similar data are available for humans (36).

\section{CONCLUSION}

In summary, the present study confirms previous experimental observations that adjuvant therapy with dexamethasone for bacterial meningitis may increase neuronal damage to the hippocampus and demonstrates for the first time that this increase in neuronal loss is associated with functional deficits in learning. Important questions regarding the basic mechanisms of this observation and its clinical significance are raised by these observations. For example, adjuvant therapy of bacterial meningitis with dexamethasone has been shown to reduce severe hearing loss in children, which also might influence overall learning performance in survivors (11). The potential to preserve hearing capacity by giving dexamethasone needs to be balanced against the possibility of inducing hippocampal damage by this treatment. Future studies must carefully assess the possible effects of dexamethasone on hippocampal function in patients with meningitis. 
Acknowledgments. We thank Philipp Joss and Andreas Messerli for excellent technical support.

\section{REFERENCES}

1. Tuomanen E 1994 Modulation of inflammation in bacterial meningitis. Isr J Med Sci 30:339-341

2. Leib SL, Täuber MG 1999 Pathogenesis of bacterial meningitis. Infect Dis Clin North Am 13:527-548

3. Pfister HW, Koedel U, Paul R 1999 Acute Meningitis. Curr Infect Dis Rep 1:153-159

4. Goetghebuer T, West TE, Wermenbol V, Cadbury AL, Milligan P, Lloyd-Evans N, Adegbola RA, Mulholland EK, Greenwood BM, Weber MW 2000 Outcome of meningitis caused by Streptococcus pneumoniae and Haemophilus influenzae type b in children in The Gambia. Trop Med Int Health 5:207-213

5. Daoud AS, al-Sheyyab M, Batchoun RG, Rawashdeh MO, Nussair MM, Pugh RN 1995 Bacterial meningitis: still a cause of high mortality and severe neurological morbidity in childhood. J Trop Pediatr 41:308-310

6. Bedford H, de Louvois J, Halket S, Peckham C, Hurley R, Harvey D 2001 Meningitis in infancy in England and Wales: follow up at age 5 years. BMJ 323:533-536

7. Grimwood K, Anderson P, Anderson V, Tan L, Nolan T 2000 Twelve year outcomes following bacterial meningitis: further evidence for persisting effects. Arch Dis Child 83:111-116

8. Merkelbach S, Sittinger H, Schweizer I, Muller M 2000 Cognitive outcome after bacterial meningitis. Acta Neurol Scand 102:118-123

9. Almuneef M, Memish Z, Khan Y, Kagallwala A, Alshaalan M 1998 Childhood bacterial meningitis in Saudi Arabia. J Infect 36:157-160

10. Coyle PK 1999 Glucocorticoids in central nervous system bacterial infection. Arch Neurol 56:796-801

11. McIntyre PB, Berkey CS, King SM, Schaad UB, Kilpi T, Kanra GY, Perez CM 1997 Dexamethasone as adjunctive therapy in bacterial meningitis. A meta-analysis of randomized clinical trials since 1988. JAMA 278:925-931

12. Daoud AS, Batieha A, Al-Sheyyab M, Abuekteish F, Obeidat A, Mahafza T 1999 Lack of effectiveness of dexamethasone in neonatal bacterial meningitis. Eur J Pediatr 158:230-233

13. Schuchat A, Robinson K, Wenger JD, Harrison LH, Farley M, Reingold AL, Lefkowitz L, Perkins BA 1997 Bacterial meningitis in the United States in 1995. Active Surveillance Team N Engl J Med 337:970-976

14. Thomas R, Le Tulzo Y, Bouget J, Camus C, Michelet C, Le Corre P, Bellissant E 1999 Trial of dexamethasone treatment for severe bacterial meningitis in adults. Adult Meningitis Steroid Group Intensive Care Med 25:475-480

15. Williams AJ, Nadel S 2001 Bacterial meningitis: current controversies in approaches to treatment. CNS Drugs 15:909-919

16. Nau R, Soto A, Bruck W 1999 Apoptosis of neurons in the dentate gyrus in humans suffering from bacterial meningitis. J Neuropathol Exp Neurol 58:265-274

17. Leib SL, Clements JM, Lindberg RL, Heimgartner C, Loeffler JM, Pfister LA, Täuber MG, Leppert D 2001 Inhibition of matrix metalloproteinases and tumour necrosis factor alpha converting enzyme as adjuvant therapy in pneumococcal meningitis. Brain 124:1734-1742

18. Zysk G, Bruck W, Gerber J, Bruck Y, Prange HW, Nau R 1996 Anti-inflammatory treatment influences neuronal apoptotic cell death in the dentate gyrus in experimental pneumococcal meningitis. J Neuropathol Exp Neurol 55:722-728
19. Loeffler JM, Ringer R, Hablutzel M, Tauber MG, Leib SL 2001 The free radical scavenger alpha-phenyl-tert-butyl nitrone aggravates hippocampal apoptosis and learning deficits in experimental pneumococcal meningitis. J Infect Dis 183:247-252

20. Leib SL, Kim YS, Ferriero DM, Täuber MG 1996 Neuroprotective effect of excitatory amino acid antagonist kynurenic acid in experimental bacterial meningitis. J Infect Dis 173:166-171

21. Scoville WB, Milner B 2000 Loss of recent memory after bilateral hippocampal lesions. 1957. J Neuropsychiatry Clin Neurosci 12:103-113

22. Morris RG, Garrud P, Rawlins JN, O'Keefe J 1982 Place navigation impaired in rats with hippocampal lesions. Nature 297:681-683

23. Wellmer A, Noeske C, Gerber J, Munzel U, Nau R 2000 Spatial memory and learning deficits after experimental pneumococcal meningitis in mice. Neurosci Lett 296:137140

24. Pfister LA, Tureen JH, Shaw S, Christen S, Ferriero DM, Tauber MG, Leib SL 2000 Endothelin inhibition improves cerebral blood flow and is neuroprotective in pneumococcal meningitis. Ann Neurol 47:329-335

25. Auer M, Pfister LA, Leppert D, Tauber MG, Leib SL 2000 Effects of clinically used antioxidants in experimental pneumococcal meningitis. J Infect Dis 182:347-350

26. Flagel SB, Vazquez DM, Watson SJ, Jr., Neal CR, Jr 2002 Effects of tapering neonatal dexamethasone on rat growth, neurodevelopment, and stress response. Am J Physiol Regul Integr Comp Physiol 282:R55-63

27. Kim YS, Sheldon RA, Elliott BR, Liu Q, Ferriero DM, Täuber MG 1995 Brain injury in experimental neonatal meningitis due to group B streptococci. J Neuropathol Exp Neurol 54:531-539

28. de Gans J, van de Beek D 2002 Dexamethasone in adults with bacterial meningitis. N Engl J Med 347:1549-1556

29. Tornello S, Orti E, De Nicola AF, Rainbow TC, McEwen BS 1982 Regulation of glucocorticoid receptors in brain by corticosterone treatment of adrenalectomized rats. Neuroendocrinology 35:411-417

30. Almeida OF, Conde GL, Crochemore C, Demeneix BA, Fischer D, Hassan AH, Meyer M, Holsboer F, Michaelidis TM 2000 Subtle shifts in the ratio between proand antiapoptotic molecules after activation of corticosteroid receptors decide neuronal fate. FASEB J 14:779-790

31. Haynes LE, Griffiths MR, Hyde RE, Barber DJ, Mitchell IJ 2001 Dexamethasone induces limited apoptosis and extensive sublethal damage to specific subregions of the striatum and hippocampus: implications for mood disorders. Neuroscience 104:57-69

32. Sousa N, Paula-Barbosa MM, Almeida OF 1999 Ligand and subfield specificity of corticoid-induced neuronal loss in the rat hippocampal formation. Neuroscience 89:1079-1087

33. Keenan PA, Jacobson MW, Soleymani RM, Mayes MD, Stress ME, Yaldoo DT 1996 The effect on memory of chronic prednisone treatment in patients with systemic disease. Neurology 47:1396-1402

34. Gianinazzi C, Grandgirard S, Imboden H, Egger L, Meli DN, Bifrare YD, Joss PC, Täuber MG, Borner C, Leib SL 2003 Caspase-3 mediates hippocampal apoptosis in pneumococcal meningitis. Acta Neuropathol (Berl) 105:499-507

35. Braun JS, Novak R, Herzog KH, Bodner SM, Cleveland JL, Tuomanen EI 1999 Neuroprotection by a caspase inhibitor in acute bacterial meningitis. Nat Med 5:298-302

36. Hassan AH, von Rosenstiel P, Patchev VK, Holsboer F, Almeida OF 1996 Exacerbation of apoptosis in the dentate gyrus of the aged rat by dexamethasone and the protective role of corticosterone. Exp Neurol 140:43-52 